\title{
Radiological/Nuclear Challenge Agent
}

National Cancer Institute

\section{Source}

National Cancer Institute. Radiological/Nuclear Challenge Agent. NCI Thesaurus. Code C158327.

A challenge agent that emits ionizing radiation. 